\title{
Giant Cell Tumor Arising from Fibular Head in Immature Skeleton Treated with Marginal Excision
}

\author{
Suresh Pandey ${ }^{1}$ \\ ${ }^{1}$ Department of Orthopaedics, Chitwan Hospital, Bharatpur-10, Chitwan, Nepal.
}

\begin{abstract}
Giant Cell tumor (GCT) is the benign tumor commonly found in second to fourth decade of life. Proximal fibula is very rare site and its occurrence in immature skeleton is still rare with very limited case report. We present a case of painful bony lesion arising from proximal fibula diagnosed as GCT in 15 year boy which was managed successfully with marginal excision and lateral collateral ligament anchorage in proximal tibia. There is no evidence of local recurrence, lung metastasis, knee pain and instability till 24 months of follow up.
\end{abstract}

Keywords: giant cell tumor; proximal fibula; marginal excision.

\section{INTRODUCTION}

Giant Cell Tumor is common benign bone tumor constituting $4-7 \%$ of all bone tumors and upto $22 \%$ of benign bone tumors. ${ }^{1,2}$ It is generally found at end of long bones in mature skeleton with peak incidence in second to fourth decade. ${ }^{3}$ Proximal tibia, distal femur and distal radius are the most common sites in order of prevalence. Proximal fibula is very rare site and occurrence in immature skeleton is still rare. We share our experience of diagnosing GCT in proximal fibula in 15 year-old boy because of its rarity and treated with marginal excision with lateral collateral ligament reconstruction by anchoring it in lateral part of proximal tibia. There is no evidence of local recurrence, pain or instability or lung metastasis till 2 years of follow up. Informed consent was taken regarding publication of data concerned.

\section{CASE REPORT}

A 15 year young boy presented with diffuse mild and constant pain over lateral aspect of right knee for 2 month. There is preceding history of playground injury with diffuse and mild knee pain and swelling 2 month ago which subsided with analgesics and rest. But constant pain localized to outer and lower aspect of the knee joint persisted. There is no history of fever, limping, instability, locking or any constitutional symptoms. On examination, there was mild and localized fullness over fibular head region right with tenderness. There was no change in color or raised local temperature (Figure 1a). Ligaments and meniscal tear tests were negative. Xray right knee AP and Lateral view showed doubtful, ill defined cystic area (Figure 1b). Haematological and Biochemical

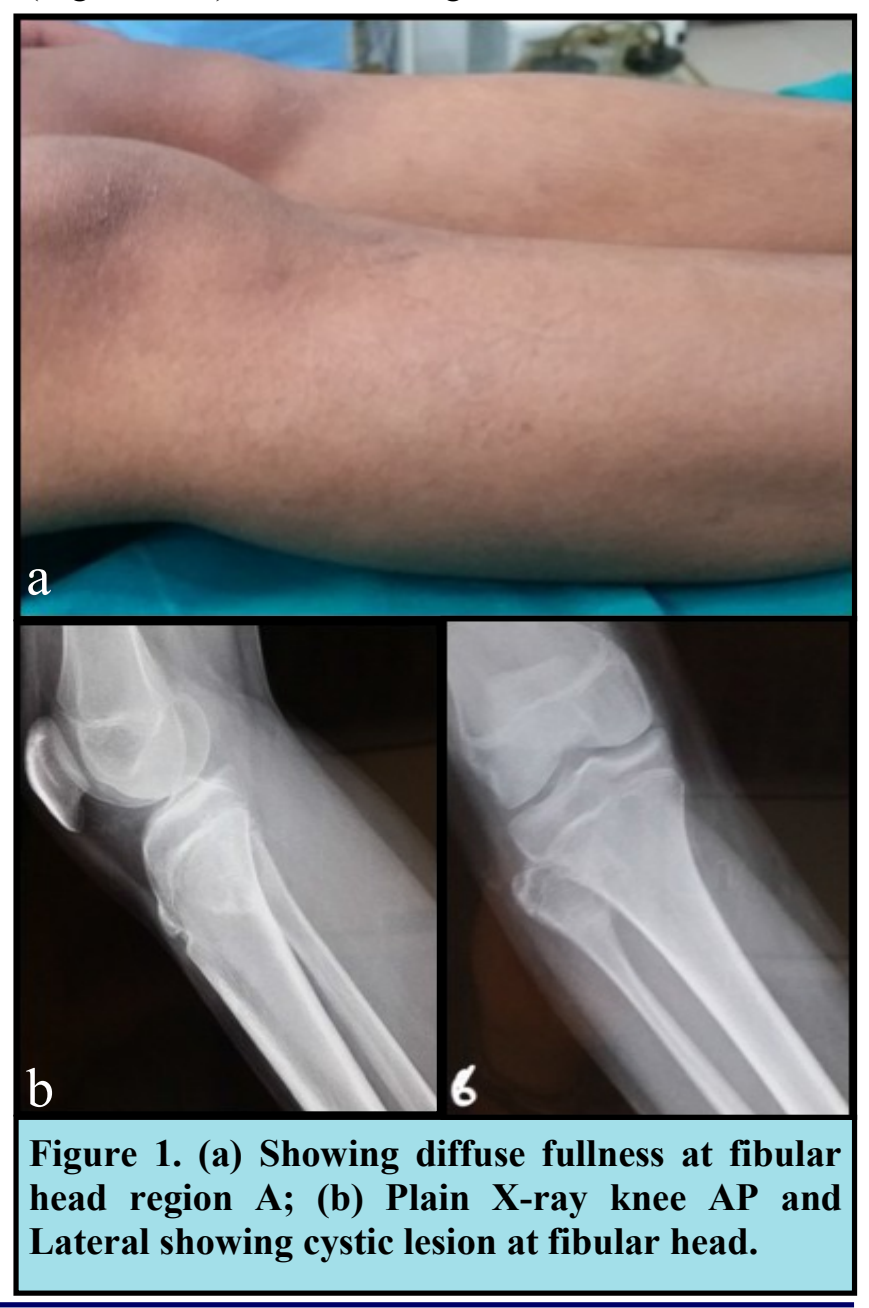

Correspondence: Dr. Suresh Pandey, Department of Orthopaedics, Chitwan Hospital, Bharatpur-10, Chitwan, Nepal. Email: drsuresh.orthonepal@gmail.com. Phone: +977-9845047228. DOI: 10.3126/ jcmsn.v14i3.20962 . Article received: 2018-09-03. Article accepted: 2018-09-16. 
parameters were not significant. As pain was constant after stopping analgesics, he underwent MRI scan right knee which showed cystic lesion extending over epi-metaphysis of fibular head without evidence of periosteal reaction, pathological fracture or any soft tissue extension suggestive of Giant CellTumor with differential diagnosis of Chondroblastoma (Figure 2). After

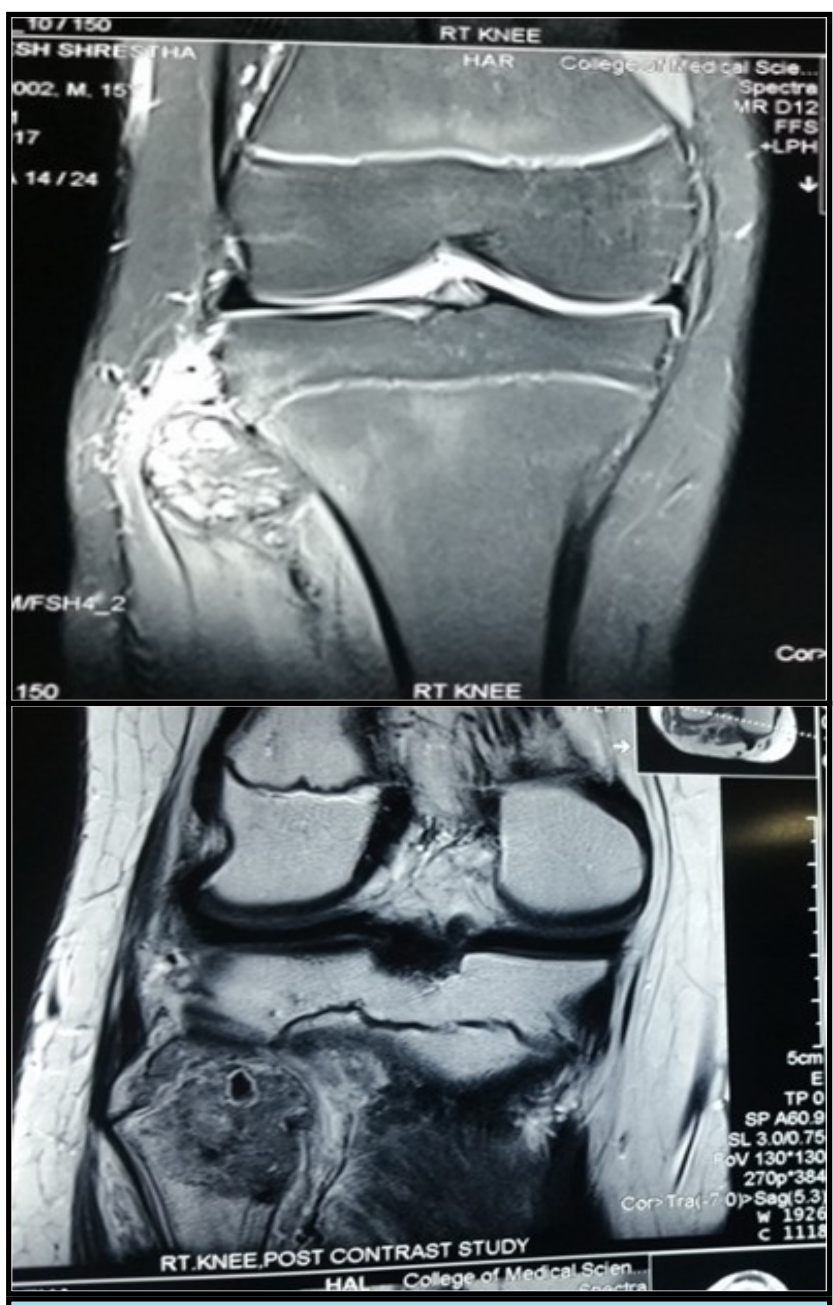

Figure 2. MRI showing epiphyso-metaphyseal bony lesion at fibular head without soft tissue extension .

explaining disease nature, treatment plan and prognosis, patient's guardian consented for operation with excision of fibular head instead of extended curettage to avoid chance of local recurrence. He was treated with marginal excision of fibular head and neck under spinal anaesthesia on lateral position with incision made over the fibular head and neck protecting common peroneal nerve. Lateral collateral ligament and Biceps femoris tendon was anchored together to bone in proximal tibia with braided nonabsorbable suture. Patient was kept on knee brace for 2 weeks and partial weight bearing crutch walking for 2 weeks.
Suture was removed at 2 weeks and progressive range of motion exercise and weight bearing walking permitted. Histopathological report showed numerous multinucleated giant cells scattered among oval to spindle cells without any atypia or mitotic figures confirming the diagnosis of GCT fibular head. There was complete relief of pain at 6 weeks with full ROM and painless full weight bearing walking at 6 weeks. There was no ligament instability on varus stress after 3 months. He has returned to running, football and other sports activities without pain or instability. There is no evidence of local recurrence or lung metastasis till 24 months of follow up.

\section{DISCUSSION}

GCT is generally found in mature skeleton and its occurrence before physeal closure is very rare. The most common sites are distal femur, proximal tibia and distal radius in order of its frequency. ${ }^{4}$ They typically arise from epyphysis and extend upto subchondral bone. It rarely involves capsule and joints. Different studies have shown GCT in immature skeletons representing $0.5 \%-10.6 \%$ of total GCTs. ${ }^{1,2}$ Ninety percent of the tumors are found in long bones and smaller bones like hand and foot, skull, vertebrae and pelvis are rare sites. ${ }^{1}$ Picci et al., have reported 6 cases of GCTs in children all around knees. ${ }^{5}$

Pain and swelling are the most common mode of presentation as in adult and pain is variable initially. Pain becomes constant once size of the tumor increases. Weakness, restricted joint motion and pathologic fracture are rare presentation. ${ }^{6}$ Course of the disease and behavior in children is same as adult with equal chance of local recurrence or occasional lung metastasis.

Literature has shown local recurrence rate $14-25 \%$ after different methods of extended curettage. ${ }^{7}$ Recurrence rate is more in Campanacci grade III and is better treated with excision if part is expendable or excision and reconstruction with different methods like autograft, tumor prosthesis, arthrodesis or allograft. Treatment of GCTs in immature skeleton is same as in mature skeleton with similar rate of recurrence. We treated this case with marginal excision of fibular head and neck as part was expendable and patient party opted for it after explaining chance of local recurrence after intralesional curettage. Lateral collateral ligament and biceps femoris muscle was taken together with nonabsorbable braided suture, number 5 Ethibond, and anchored to the proximal tibia through drill 
tunnel. There is variable result and evidence about the lateral knee instability after lateral collateral ligament leaving as such after tumor excision or anchoring to proximal tibia. There was no evidence of local recurrence, knee instability, restricted motion of knee, pain or lung metastasis till 24 months of follow up. There is no case report of such lesion in immature fibular head treated with excision and ligament reconstruction just by anchoring to proximal tibia with good result in our knowledge in the literature.

\section{CONCLUSION}

Local pain and diffuse swelling over fibular head region in immature skeleton should prompt us to think of GCT as one of the differential diagnosis and investigate further even if plain $\mathrm{X}$ ray looks apparently normal initially. Since part is expendable and considering high chance of local recurrence, marginal excision with lateral collateral anchorage on proximal tibia can give good result without any local or systemic complication.

\section{Conflict of interest: None}

\section{REFERENCES}

1. Campanacci M, Giunti A, Olmi R. Metaphyseal and diaphyseal localization of giant cell tumors. La Chirurgia Degli Organi di Movimento, vol. 62, no. 1, pp. 29-34, 1975.

2. Unni KK, Inwards CY. Dahlin's Bone Tumors: General Aspects and Data on 10,165 Cases, Lippincott Williams and Wilkins, 2010.

3. Jaffe HF. Tumors and Tumorous Conditions of the Bones and Joints. Lea and Febiger. Philadelphia, Pa, USA, 1958.

4. Bridge JA, Neff JR, Mouron BJ. Giant cell tumor of bone. Chromosomal analysis of 48 specimens and review of the literature. Cancer Genet Cytogenet. 1992;58(1):2-13.

5. Picci P, Manfrini M, Zucchi V. Giant-cell tumor of bone in skeletally immature patients. The Journal of Bone \& Joint Surgery-American Volume. 1983;65(4):486-490.

6. Turcotte RE. Giant cell tumor of bone. Orthopedic Clinics of North America. 2006;37 (1):35-51.

7. Turcotte RE, Wunder JS, Isler MH. Giant cell tumor of long bone: a Canadian Sarcoma Group study," Clinical Orthopaedics and Related Research. 2002;397:248-258.

Citation: Pandey S. Giant Cell Tumor Arising from Fibular Head in Immature Skeleton Treated with Marginal Excision. JCMS Nepal. 2018;14(3):170-2. 